\title{
Comprensión del vínculo afectivo de madres adolescentes con sus hijos en edades de 0 a 5 años
}

\section{Understanding the affective bond between teenage mothers and their children between 0 and 5 years old}

\author{
Katine Buelvas-Sanjuan. \\ Fundación mi alegra infancia. Coordinación. Barranquilla, Colombia. \\ $\triangle$ mialegreinfanciabq@gmail.com \\ (D) https://orcid.org/0000-0002-4283-2476
}

Ernesto-José Cortez-Soto.

Corporación Universitaria Minuto de Dios. Administración de Empresa, Santa Marta, Colombia.

$\square$ ernesto.cortez@uniminuto.edu.co

(iD) https://orcid.org/0000-0003-2329-0912

David Borge-Donado.

Ceipa Business School. Ciencias Administrativas. Medellín. Colombia.

$\triangle$ david borgedo@virtual.ceipa.edu.co

(i) https://orcid.org/0000-0002-6952-3315

Recibido: 02-10-2019

Aceptado: $14-12-2019$

\section{RESUMEN}

Se presentan los resultados de una investigación cualitativa acerca de la comprensión del vínculo afectivo entre madres adolescentes con sus hijos de edades entre los 0 a 5 años. Se realizó una investigación de tipo cualitativo, enmarcada en el método fenomenológicohermenéutico, recolectando datos mediante dos grupos focales y cuatro entrevistas a profundidad con tres participantes. El principal objetivo fue conocer el significado que le dan las madres adolescentes a sus formas de relacionarse y establecer vínculos con sus hijos de edades entre los cero y cinco años. Y, a través de ello, comprender el significado que le dan a su experiencia de ser madres adolescentes; la manera en que interactúan con sus niños y niñas; conocer los valores que reafirman o evitan en sus hijos; conocer las dinámicas de cuidado y bienestar que prevalecen en las relaciones; y el rol que desempeñan, en este proceso, diferentes actores, como abuelas, padres, parejas, pares y sociedad.

Palabras claves: Vínculo afectivo; madres adolescentes; niños; dinámicas de cuidado

\section{ABSTRACT}

The results of a qualitative research about the understanding of the emotional bond between teenage mothers and their children between the ages of 0 and 5 are presented. A qualitative research was carried out, framed in the phenomenological-hermeneutical method, collecting data through two focus groups and four in-depth interviews with 
three participants. The main objective was to know the meaning that teenage mothers give to their ways of relating and establish links with their children between the ages of zero and five. And, through it, to understand the meaning they give to their experience of being teenage mothers; the way they interact with their children; to know the values that reaffirm or avoid in their children; to know the dynamics of care and well-being that prevail in relationships; and the role played by different actors in this process, such as grandmothers, parents, couples, peers and society.

Keywords: Affective bond; teenage mothers; children; care dynamics

\section{INTRODUCCIÓN}

Tener embarazos en la adolescencia son casos muy comunes en los países subdesarrollados, sobre todo en los estratos socioeconómicos bajos. Según estadísticas, para el caso de Colombia, en los últimos años este fenómeno ha disminuido. Sin embargo, sigue siendo elevado el número de casos reportados. Según el Departamento Administrativo Nacional de Estadística (DANE, 2015), el 20,5 \% de las mujeres con hijos en el país, los tuvieron entre los 15 y los 19 años de edad. Esto, según Profamilia (2010), en Colombia ha estado caracterizado por el inicio a temprana edad de la actividad sexual, estableciendo que tres de cada cinco mujeres a los 19 años ya han sostenido relaciones sexuales, puntualizando que aquellas mujeres sin grados de escolaridad inician su vida sexual cinco años antes. Además, se establece que el 19\% del total de mujeres adolescentes ya son madres o esperan su primer hijo, lo que se asume en la medida en que una de cada cinco mujeres ha estado embarazada alguna vez a los 17 años y una de cada tres a los 19 años. Es de anotar que estas cifras aumentan en las zonas rurales periféricas y dispersas, en donde el $26 \%$ de las mujeres ha estado en embarazo o espera un hijo a los 17 años. En la ciudad de Barranquilla, según la Secretaría de Salud (2015), se registra un $19 \%$ de embarazos adolescentes y que estos se dan o prevalecen en zonas de estratos socioeconómicos bajos.

La presente investigación tuvo como objetivo estudiar y comprender el vínculo afectivo que se establece entre madres adolescentes e hijos, partiendo de que el primer escenario de interacción que tiene el niño o la niña es el recibimiento que se le brinda a la llegada a la sociedad, el cual permitirá a los niños y niñas sentirse seguros y en condiciones para llevar a cabo procesos de exploración, regular sus emociones e interactuar con otras personas de su medio.

Al realizar revisiones bibliográficas, el tema de embarazos adolescentes se encuentra en muchos estudios e investigaciones. Sin embargo, predominan estudios de tipo cuantitativo. Desde perspectivas de factores de riesgos para la salud en la madre y sus hijos, prevalencia de nacimiento de niños con malformaciones, bajo peso, morbilidad y mortalidad neonatal; $y$, desde la perspectiva social, se encuentran investigaciones en temas como transmisión de la pobreza, y predominan estudios de tipo cuantitativo. Se encuentran pocas investigaciones acerca de la generación de vínculos afectivo y apego en investigaciones de tipo cualitativo.

El desarrollo de relaciones tempranas en los niños se precisa como de gran relevancia para el desarrollo posterior de relaciones sociales. En ese sentido, Ortega, Rodríguez \& 
Jimenez (2013) refieren que un alto grado de la formación de los niños y adolescentes corresponde a la esencia del núcleo familiar y a la corresponsabilidad paternal en educar, criar y orientar a los hijos en la manera de interactuar, cuidar, relacionarse y fundamentar diversas normas y pautas que conciben su pensamiento y comportamiento, dando lugar al desarrollo integral y psicológico de los mismos, así como el de su expresión en cada etapa de crecimiento y desarrollo.

La base de estas formas iniciales de relacionarse o acercarse a otro individuo nace en la primera relación madre-hijo. Gago (2013) introduce la Teoría del Apego de Bowlby, en la que este se establece como una relación en la que se desarrolla un vínculo afectivo caracterizado o tendiente a buscar contacto y proximidad en una figura cercana y mayor al niño o adolescente. A partir de lo anterior, se desarrollan una serie de conductas que definen el tipo de apego y se refieren a: i) Conductas de Apego, que hacen referencia a las intenciones y demandas de esa relación; ii) Sentimientos de Apego, que son las experiencias afectivas referidas con sentimientos hacia la figura de apego y iii) Representación Mental, que es la forma en que los niños internalizan el tipo de relación con la figura de apego en sentimientos, dependencia, cuidado, etc. De igual forma, se clasifican los estilos de apego en: i) Seguro, ii) Inseguro Evitativo y iii) Inseguro Ambivalente. Agregando a esta clasificación el Apego Desorganizado-Desorientado, definido en la actualidad, en la llamada Era Representacional, por Mary Main \& Salomón (1986).

Partiendo de lo anterior, es necesario comprender que la adolescencia, como etapa, comprende una época de transición hacia la adultez. Por tanto, los adolescentes comienzan a experimentar nociones de madurez e inmadurez en la toma de decisiones, intentarán sincronizar sus vidas a su parecer y creerán mantener todo en orden, según sus conocimientos, por lo cual son muy propensos a ocultar cosas o inventar otras para satisfacer sus necesidades de ego o aceptación en otros grupos. Cabe resaltar que los adolescentes no poseen los elementos suficientes para la toma de decisiones sobre responsabilidad laboral, sexual o social, por lo cual su ritmo de vida es retraído, al 100\% en algunos casos o netamente extrovertido, por lo cual se considera como una etapa de transición inestable, puesto que, en ella, inciden muchos aspectos relacionados con la familia, la aceptación, la autoestima, entre otros, que definen las actitudes y la toma de decisiones de los jóvenes (Escuela de Familia Moderna, 2013). En términos generales, un estadio de la vida humana intermedio entre la infancia y la edad adulta.

Siguiendo a Erikson (1973) y su propuesta de las ocho etapas del desarrollo del ego para comprensión de la adolescencia, nos centraremos en las dos que hacen referencia a la adolescencia en tanto tal, las cuales son la Etapa Quinta (de los 12 hasta los 18 años) y la Etapa Sexta (la Juventud, de los 18 a los 24 años). Erikson define la adolescencia como ese paréntesis que existe entre la niñez y la vida adulta, una etapa psicológica necesaria, en la que se van dando bases, tiempo y energía para los nuevos roles que se deben desempeñar; el adolescente empieza a vivir con autoimágenes distintas. Según Erikson, existe una Quinta Etapa, en la que se da una crisis del yo, se vivencia el dilema Identidad del Ego vs Confusiones del Rol. En esta etapa, el individuo va formando su autoimagen; se experimenta ansiedad y temores por el descubrimiento de intereses y creación de la autoimagen. En este proceso de construcción se le da gran valor a la imagen u opinión que tienen otros de él. En esta etapa se va construyendo una imagen propia a partir de la integración de ideas propias, de quienes lo rodean y de lo que se 
quiere alcanzar; lo ideal en esta etapa es lograr una imagen coherente y congruente, ya que, de lo contrario, traerá consecuencias como una crisis de identidad.

Erik Erikson propone la llamada Sexta Etapa, que va desde los 18 a los 24 años. Esta etapa está enmarcada en el dilema Intimidad vs Aislamiento. Uno de los principales aspectos que se logra es la Independencia con relación a la familia y a los lugares e instituciones que actúan en roles similares.

La intimidad es reconocida como la capacidad de relacionarse de forma cercana con otra u otras personas, con un gran sentido de interés y compromiso, a partir de amistades cercanas y relaciones sexuales, sentimientos de interés y compromiso.

\section{Etapas del Desarrollo en la Primera Infancia}

Se toma la concepción y el análisis de las etapas propuestas por Erikson (1973) para la primera infancia, las cuales son las tres primeras de sus ocho etapas propuestas para el desarrollo psicosocial y las fortalezas básicas en cada una de ellas.

Primera Etapa Oral-Sensorial. Se reconoce desde el momento del nacimiento hasta el primer año. La boca es de gran importancia en esta etapa, ya que, a través de ella, tiene contacto con su madre, experimenta, vivencia y descubre sensaciones. El dilema de esta etapa está enmarcado en Confianza vs Desconfianza, la cual se define de acuerdo con lo que reciba del medio y de quienes lo rodean. De la manera como recibe afecto y la capacidad de reacción ante la satisfacción de sus necesidades, se convierten en aspectos determinantes en el desarrollo de la confianza o desconfianza.

Segunda Etapa Anal-Muscular. Comprendida desde el primer año de vida hasta los tres años. El dilema de esta etapa es Autonomía vs Vergüenza y Duda. Los niños o niñas ven su Yo como una persona con derechos propios; se reconocen separados de los padres, al poder realizar muchas acciones por sí solos, pero reconocer cierta dependencia hacia ellos. En este momento, toma gran valor la importancia y oportunidades que dan los adultos a la libre expresión de los niños y niñas. La gran dificultad se presenta cuando los adultos obstruyen la libre expresión y empiezan a establecer normas sociales, como lugares apropiados para determinado comportamiento.

Tercera Etapa Genital-Locomotora. Comprendida desde los tres años de vida hasta los cinco años. Esta etapa se caracteriza por la admiración o deseo de total atención del padre del sexo opuesto, el llamado Complejo de Edipo, y de "competencia" con el padre del mismo sexo. La iniciativa de los niños, en esta edad, debe ser canalizada por los adulos que los acompañan en el proceso hacia metas realistas y con aprobación del contexto en el que se desarrollan; en este momento se desarrolla el llamado "súper yo". El dilema de esta etapa es Iniciativa vs Culpa. El niño o niñas desarrolla una gran imaginación, imita a los adultos y logra prever roles. Si no se logra desarrollar esta iniciativa se mostrará cómo un poco espontaneo, manifestará muchos celos, se mostrará desconfiado y evasivo. Se desarrolla la fortaleza básica del propósito, que se deriva de la iniciativa; es una manera de demostrar coraje e interés por seguir procesos para el cumplimento de metas que se trazan. 


\section{MÉTODO}

El desarrollo metodológico de esta investigación se fundamentó en el método Fenomenológico-Hermenéutico. Por ello, se describió y buscó comprender el vínculo afectivo de las madres adolescentes con sus hijos en edades entre los 0 a 5 años. Se inició con los relatos detallados de madres adolescentes y se complementó con una clarificación de preconcepciones, predisposiciones o prejuicios del investigador, por medio de un método que integre la fenomenología y la hermenéutica. Esta integración se realizó teniendo en cuenta una variación del método de Giorgi (2003).

La integración de la hermenéutica y la fenomenología, como métodos de investigación, permiten que - a través del estudio de situaciones vividas, experiencias y/o percepcionesse llegue, a partir del estudio particular de los casos, a posibles generalidades, y organizarlos de tal manera que sean reconocidos y presentados como teorías o conocimiento científico. Esta integración permitió la construcción de una interpretación acertada y coherente con la realidad de la participante, hasta lograr encontrar un significado real del fenómeno estudiado. A partir de ello (De Castro, 2003-2005), el método fenomenológico-hermenéutico, se contextualiza a partir de una concepción y entendimiento existencial del ser humano, lo cual implica ciertas variaciones al método fenomenológico aplicado por Giorgi. Siguiendo a De Castro (2005) y Garza (2004) esta investigación se dio en tres etapas y cada una de ellas se dividió en diferentes momentos: Etapa Descriptiva, subdividida en clarificación de presupuestos, definición de preguntas orientadoras, elección técnica y elaboración de la descripción protocolaria por parte de los coinvestigadores; una segunda etapa, la Estructural, subdividida a su vez el momento de familiarización con la experiencia de la participante, delimitación de unidades de sentido, tematización, transformación, clasificación de propias interpretaciones y/o prejuicios y determinación de temas para el próximo encuentro; finalmente, una tercera etapa en la que se dio la integración de experiencia, determinación de divergencias y convergencias, estructura del sentido, retroalimentación y conclusiones.

Esta investigación es de tipo cualitativo. Se realizó con descripciones protocolares, dos grupos focales de 7 adolescentes cada uno y cuatro sesiones de entrevistas a profundidad en forma de test con tres participantes.

\section{RESULTADOS}

A partir de la dinamización planteada con los grupos focales y las entrevistas en profundidad, se dio claridad a algunas ideas, temáticas y aspectos, que no fueron posibles por la misma naturaleza de la dinámica del grupo focal. La información obtenida es de gran relevancia, ya que esas jóvenes coinvestigadoras estaban vivenciando la situación; por lo tanto, es un escenario perfecto para conocer la forma cómo lo afrontan, y las emociones que surgen frente a esta vivencia fueron propicias para comprender cómo se da el vínculo madre-hijo durante los primeros años de vida, en un contexto situacional como es el embarazo adolescente.

De los resultados de la aplicación de los grupos focales y las entrevistas en profundidad, con base en las preguntas orientadoras, surgieron cuatro (4) categorías y doce (12) subcategorías, las cuales se organizaron de la siguiente manera: 
Tabla 1. Relación Categorías y Subcategorías

\begin{tabular}{|c|c|}
\hline Categoría & Subcategoría \\
\hline & Emociones frente embarazo \\
\hline \multirow[t]{3}{*}{ Concepción del embarazo adolescente } & Contexto situacional \\
\hline & Implicaciones del embarazo \\
\hline & Valor otorgado a su hijo \\
\hline \multirow[t]{3}{*}{ Vínculo madre adolescente- hijo } & Formas de cuidado y percepción de bienestar del bebé \\
\hline & Expectativas para la crianza y valores que se reafirman o evitan \\
\hline & Rol de los padres o figuras maternales (abuelos del bebé) \\
\hline \multirow[t]{3}{*}{ Vínculos con familia y figuras de apoyo en la nueva etapa } & Rol y apoyo de la pareja (Padre) \\
\hline & Dependencia madre adolescente y familia o figura de apoyo \\
\hline & Percepción de la comunidad hacia la adolescente en su nuevo rol \\
\hline \multirow[t]{2}{*}{ Relaciones sociales } & Figuras de apoyo en la comunidad \\
\hline & Relaciones con pares \\
\hline
\end{tabular}

\section{Concepción del Embarazo Adolescente}

Emociones frente al Embarazo. Frente a las percepciones sobre el estado de embarazo, se encontró que sienten miedo, preocupación y se pusieron muy nerviosas cuando conocieron la noticia, ya que no habían considerado la posibilidad de quedar embarazadas y tener un hijo a su edad, y son conscientes de los cambios que puede traer esta nueva etapa, tanto a nivel de salud como de responsabilidades adquiridas en el nuevo rol. Las emociones que sienten son muy confusas, ya que, de manera inmediata, piensan en la reacción que tendrán sus padres, los cambios en sus vidas, y le temen a estar solas enfrentando esta nueva situación.

En primera instancia, se niegan a aceptar y a creer su nueva realidad; no se sienten preparadas ni fisiológicamente, ni emocionalmente. La primera actitud, pensamiento o posición es rechazar el embarazo y desear no tener ese hijo. Sin embargo, no materializan la idea, ya que no recurren a ningún método o estrategia para interrumpir su embarazo, lo cual es soportado en los relatos de algunas de las participantes "Pues sentí miedo, porque todavía no estaba apta para un embarazo y tampoco para un parto, pero gracias a Dios me ha ido bien"

Esto se puede interpretar en un estudio desarrollado por Mazuera, Trejos \& Reyes (2017), quienes encuentran que la experiencia del embarazo adolescente se enmarca en una etapa inicial de problemas emocionales, arrepentimientos y sufrimiento dada por la connotación familiar representada en el embarazo como un problema, el no poder continuar con la vida escolar, los problemas de inserción laboral, el señalamiento público y el aumento de la pobreza como factor elemental para el mantenimiento de la vida.

Contexto Situacional. Las madres adolescentes tomaron conciencia de su embarazo en etapas adelantadas y, por ende, empezaron controles médicos y cuidados pertinentes 
en periodos avanzados del embarazo. La primera etapa estuvo enmarcada por un periodo de negación frente al hecho de estar embarazadas, aún con indicios como relaciones sexuales sin protección; ausencia de periodo menstrual; síntomas físicos, como vómitos o náuseas; sospechas de sus familias o pares; y que no consideraban la posibilidad de estar en estado de embarazo, ya que, en sus fantasías, no creían estar aptas para un embarazo.

La mayoría de las adolescentes establecen que su dinámica juvenil se desarrollaba normal, mayoritariamente en sus hogares, colegios y en ocasiones en los hogares de amigos cercanos, y que conocieron a los padres de sus hijos en esos contextos, fueron la primera y única persona con quien han tenido relaciones sexuales y no había planes de tener hijos.

En los contextos familiares se evidencia mayor cercanía con las abuelas, a quienes, de cierta manera, reconocen como salvadoras o apoyos en medio de todas las dificultades o diferencias familiares de las que se sienten víctimas.

Implicaciones del Embarazo. Para las adolescentes, el embarazo implica cambios en sus roles y en los planes que tenían de terminar el colegio, seguir estudiando y alcanzar su autonomía para no depender económicamente de su familia. (S) "Ahora me tocaría estudiar; mi tío me dijo que ahora que tuviera el cartón de bachiller me ayudaba con un trabajo en cualquier centro comercial o algo, porque él tiene palancas, pero siempre me ha gustado la medicina, entonces ya veo como el obstáculo ahí..."

Sobre esto, Vélez (2012) profundiza en que en la etapa adolescente resulta difícil encontrar una sintonía o compatibilidad de la situación de embarazo con la forma de crianza, educación y hasta de inserción laboral, puesto que la reproducción adolescente no se asume desde un rol o cualidad de autonomía económica. En ese sentido, el sentir que deben ser responsables las conlleva a intentar definir o estabilizar el tipo de relación que tenían con su pareja, lo cual significa, en algunos casos; retomar una relación que ya había terminado; tomar la decisión de irse a vivir juntos; formalizar una relación, cuando la pareja responde de manera positiva al embarazo; o alejarse de forma definitiva, cuando lo consideren necesario, y seguir el camino de la maternidad sola.

\section{Vínculo Madre Adolescente-Hijo}

Valor otorgado a su Hijo. Las madres adolescentes perciben a sus hijos como una compañía para siempre; los perciben como un apoyo incondicional; los consideran realmente su familia y como una oportunidad de construir un tipo de familia nueva. Manifiestan un gran sentimiento hacia ellos y lo argumentan reconociendo todos los sacrificios y esfuerzos que han hecho por ellos.

(K) "es maravilloso, es tener acompañante para no andar todo el tiempo sola"

Los hijos son vistos como una nueva brújula o motor para continuar con su vida y no desfallecer en los momentos de adversidad. Después del parto, se hace más fuerte el vínculo y la relación físico-emocional de madre e hijo; aunque en el embarazo sentían afecto, reconocen que al principio no era tan fuerte. De hecho, alguna de las coinvestigadoras reconoció sentir rechazo hacia su hijo durante el embarazo y los primeros meses de nacido, al percibirlo como una competencia o culpable de su situación de pareja 
deteriorada. Se evidencia que a las madres les cuesta definir con palabras lo que sienten por ellos, ya que sienten que es algo muy puro e indescriptible, que se intensificó desde el momento que los pusieron en su pecho y conocieron. Después de ese momento, el sentimiento va aumentando, hasta el punto de considerar que no se podrían separar de ellos. Les da temor que alguien se los quite o que algo les impida estar con ellos. En ese plano, Echevarría (2015) resalta que una de las principales consecuencias del coctel de emociones resultante de la etapa de embarazo y su posterior parto, son los problemas relacionados con la depresión, que se presenta por: i) Sobrecarga emocional de la persona por la situación familiar; ii) Falta de orientación para criar y cuidar del hijo durante el primer año; y iii) Los problemas económicos al no sostener una estabilidad financiera o laboral.

Formas de Cuidado y Percepción de Bienestar de sus Hijos. Las madres adolescentes consideran que cuidar y garantizar los cuidados de un hijo es una responsabilidad muy grande; sienten que si fueran adultas podrían enfrentarlo de mejor manera; se consideran inexpertas, torpes y temerosas frente a temas de higiene, cuidado personal, alimentación del bebé e identificación de signos de alarmas ante enfermedades.

Las formas de cuidado y percepción de bienestar del bebé están direccionadas u orientadas por sus familias cercanas, quienes apoyan de manera directa todo el proceso. Las formas de cuidado y percepción del bienestar se perciben en razón del estar pendiente del estado de salud de los niños, garantizar alimentación, protección frente al peligro físico y necesidades emocionales, que se satisfacen a través del juego con ellos y el diálogo en lugar de las agresiones físicas.

Sobre el estilo de crianza que emplean las adolescentes, Peña, Villavicencio, Palacios \& Mora (2015) resaltan que la mayoría de las madres aplican los modos de crianza que recuerden cómo fueron enseñadas o el ejemplo que tuvieron en su hogar; de igual forma, si conviven con sus padres o los de la pareja, establecerán una forma de crianza que defina como usual en la familia. De forma general, en la mayoría de los casos cuando existe quiebre de relaciones de contacto con la familia, las madres adolescentes siguen patrones de crianza poco autoritarios, poco democráticos y poco permisivos, en donde el estado que más cala es el permisivo al estar consignado por la premisa de que los hijos se deben criar sin la dependencia o apego total de la madre.

Expectativas para la Crianza y Valores que se Reafirman o Evitan. Frente a las expectativas para la crianza, las madres adolescentes buscan que sus hijos la reconozcan como autoridad, que las obedezcan, por lo que se preocupan por ganarse su respeto. Sienten una profunda preocupación porque les suceda lo mismo que a ellas. Reconocen la importancia del diálogo y evitan los gritos o los malos tratos; sin embargo, algunas veces sienten que se salen de control, alzan la voz y los amenazan con pegarles.

Frente a lo anterior, manifiestan el deseo de criar a sus hijos bajo sus propios criterios, pero se enfrentan constantemente a que sus familiares más cercanos como madres, abuelas y suegras intervienen en la crianza con acciones que en ocasiones son contrarias a las suyas; esto se presenta, según Coronado (2013) afirma, porque las madres adolescentes le dan resignificaciones a la disciplina con las que fueron educadas, y hacen, de manera consciente, cambios de estrategias en la manera de sancionar o castigar a sus hijos, por lo que prefieren el diálogo y evitan el castigo físico 


\section{Vínculos con la Familia y Figuras de Apoyo en la Nueva Etapa}

Rol de los Padres o Figuras Maternales. Las madres adolescentes coinciden en la importancia del apoyo de sus abuelas, madres o suegras, durante el embarazo y posterior al nacimiento en el proceso, ya que las reconocen como un modelo en términos de cuidados y experiencia en los procesos de crianza. El vínculo con estas figuras maternales se fortalece, ya que comparten todas las horas del día con ellas y representan un apoyo contundente en la nueva etapa.

En los procesos de crianza, las madres sienten que pierden autoridad frente a los niños, ya que sus figuras maternas o suegras tienden a desautorizarlas y apoyar o celebrar comportamientos que las madres adolescentes consideran equivocados, tales como las pataletas. En el caso de los padres de familia, se evidencia que no había una buena relación antes del embarazo, la cual se agrava aún más cuando conocen la noticia: se sienten ofendidos. La primera reacción de los padres es rabia y lo demuestran a través del maltrato verbal. Duran largos periodos sin hablarse con sus hijas, se mantienen al margen de los primeros cuidados del embarazo y retiran cualquier tipo de apoyo. Frente a lo anterior Carrillo, Maldonado, Saldarriaga, Vega \& Diaz (2004) afirman que:

El papel de los abuelos, como figuras de apego, es especialmente relevante en el caso de las madres adolescentes, ya que una de las principales características de la maternidad a esta edad es que se enmarca dentro de lo que algunos autores denominan sistemas familiares de tres generaciones, conformados por abuelos, madres adolescentes y bebés. (pag.411)

Rol y Apoyo de la Pareja. Las parejas de las adolescentes no tenían intención de tener un embarazo. De hecho, la mayoría no tenían una relación estable y, debido al embarazo, se vieron en la obligación de tomar una decisión de continuar o no juntos para asumir el embarazo. Las madres adolescentes perciben a sus parejas como un apoyo en el embarazo y con la crianza del niño; sin embargo, parece enfatizar el apoyo en términos de apoyo económico y respaldo financiero, ya que ellas no ganan dinero propio para sus gastos básicos y del bebé, y, de igual manera, consideran que estar con ellos disminuye la presión social .Según Nieto, Moreno, Abad, Martos \& Olalla (2012), para las madres adolescentes el apoyo percibido de la pareja es un punto de gran importancia en el proceso de racionalización del embarazo.

Dependencia de la madre adolescente hacia su familia o figura de apoyo. Las madres adolescentes asignan un gran valor a la compañía y apoyo que reciben de sus madres, abuelas y suegras; sienten que sin ellas no podrían haber salido adelante con el embarazo y con los cuidados de sus hijos durante los primeros meses. Vélez \& Figueredo (2015) afirman que la dependencia emocional y financiera hacia las figuras de apoyo es la consecuencia de tener que asumir un nuevo y complejo rol, como el ser madres y haber completado las etapas de la adolescencia. El enfrentar esta compleja etapa, genera en la adolescente tensión y consecuencias negativas emocionales y psicosociales, además de la dependencia económica y emocional de su red de apoyo familiar, y se ve enfrentada al conflicto generacional que esto conlleva. 


\section{Relaciones Sociales}

Percepción, según la Madre Adolescente, de la Comunidad hacia Ella en su Nuevo Rol. Las madres adolescentes sienten que la comunidad las percibe de manera distinta; consideran que, a partir de su nuevo rol, la comunidad no les da el mismo valor; sienten que el concepto de ellas se ha visto afectado de forma negativa a partir de su embarazo. Manifiestan que se sienten agredidas y desprestigiadas.

Según Nieto et. al. (2012), las madres adolescentes perciben críticas negativas; sin embargo, no es considerado como un problema grave, siempre y cuando sientan la aceptación y apoyo de su red más cercana.

Figuras de Apoyo en la Comunidad. Las madres adolescentes le otorgan mucho valor las figuras de apoyo que emergen en la comunidad: son pocas y convierten en personas muy importantes para ellas, ya que consideran que las apoyan desinteresadamente, aunque no sean de su familia. Valoran que no las juzgan y que, por el contrario, se muestran solidarias; les atribuyen el sentirse esperanzadas y valoradas, y, en la mayoría de los casos, tienen historias de vidas similares.

En ese sentido, crean condiciones sobre personas que no las critiquen tendientes hacia una relación positiva, principalmente destacado como un nuevo orden de relaciones y hasta un nuevo núcleo familiar, sobre el que sostienen sus principales procesos de apoyo y socialización. De igual forma, se establece que el desarrollo de este tipo de relaciones, que por lo general no son cuantiosas, atribuyen a la madre adolescente un criterio de percepción de que está viviendo una vida adulta, en la que las relaciones sociales de amistad e interrelación disminuyen (Bensaja et. al., 2012).

Relaciones con Pares. Los pares son quienes están con las madres adolescentes en el momento en que descubren que están embarazadas, e, incluso, quienes los alertan en torno a la posibilidad de estar embarazadas. Se encontró que, a partir del embarazo se alejan de sus pares y se deterioran las relaciones, se dedican solo a sus nuevos roles y se alejan de los espacios que compartían anteriormente, como escuela y fiestas. Se frecuentan de manera muy esporádica, como para saber cómo va el embarazo y conocer al bebé; sin embargo, toman distancia porque siguen en planes que ya ellas no pueden hacer. Ello, en razón a que el círculo de amistades se reduce en respuesta a un síntoma de perdida social que supone el nacimiento del hijo (Nieto et al., 2012).

\section{DISCUSIÓN}

A partir del análisis de las categorías se pudo reconocer que las madres adolescentes vivencian el embarazo, y la relación con sus hijos de cero a cinco años, como un proceso que genera múltiples cambios de tipo biopsicosociales, adicionales a los que ya trae consigo misma la adolescencia. A nivel general, se puede decir que predomina el miedo al desempeño que tendrán en su nuevo rol de madres; la responsabilidad de tener a otro ser humano a cargo y la falta de apoyo o el abandono de sus familias y/o parejas sentimentales, y grupos de amistad; el abandono del colegio y la interrupción de los proyectos que tenían. Se encontró, predominantemente, que durante el embarazo y los primeros meses de vida de su hijo se presenta miedo y rechazo por los cambios que 
el menor representa en sus vidas. Sin embargo, con el pasar de los meses, el vínculo afectivo se fortalece y el valor otorgado a su hijo aumenta firmemente, debido a que los consideran como algo absolutamente propio y una compañía para el resto de su vida. Se encontró que predominan situaciones en común entre las madres adolescentes, como lo es pertenecer a familias disfuncionales, carencias de cuidadores como madres o padres, ya sea por fallecimiento o abandono, relaciones difíciles y poco sanas con la familia.

En ese sentido, lo desarrollado por Bensaja et. al. (2012) se resalta en este estudio, en que muchas adolescentes asimilan la condición de embarazo como una oportunidad para establecer un escenario familiar nuevo y propio, en donde sean los determinadores de su vida, en convivencia con una pareja o con otra persona, con quien resalten un vínculo afectivo directo. De igual forma, establece dicha condición como un enlace para el mejoramiento de la situación financiera, puesto que se puede dar la premura de la obtención de trabajo; se establece un apoyo inicial por parte de la familia, un pariente o una persona o amigo muy cercano a la madre o la pareja; estableciendo un sentido de independencia "anhelado" por la adolescente, sea por las variadas circunstancias que se hayan presentado con su núcleo familiar o de la vida anterior.

Las adolescentes, cuando descubren que están embarazadas, sienten rechazo hacia su hijo, primordialmente ocasionado por el miedo a enfrentar un nuevo rol "maternal" para el que consideran no están preparadas. En el presente estudio, se reafirma lo establecido por Mazuera, Trejos \& Reyes (2017), referente a que las adolescentes consultadas destacan situaciones de rechazo y señalamiento al presentar la condición a sus familiares o al hacerse notorio el estado de embarazo, lo cual se suma a los problemas emocionales que, mentalmente, se desatan por la pérdida de la oportunidad de estudio, trabajo y de la buena relación familiar, si existe.

Uno de los principales cambios que enfrentan las jóvenes adolescentes es el abandono del colegio y las dificultades para la inserción a la vida laboral, ya que, posterior al nacimiento de su hijo, se dedican completamente su tiempo al cuidado de este; es, de cierta manera, una forma de demostrar que asumen las consecuencias de sus actos. Vélez (2012) frente a esto afirma que en la adolescencia se dificulta encontrar el equilibrio o la realización paralela de diferentes aspectos, como lo son la educación de la madre, los procesos de crianza y la inserción al mercado laboral, ya que es muy común que las madres adolescentes sigan viviendo con sus familias o suegros, y deban dedicarse al cuidado de su hijo, exclusivamente. Frente a ello, en la presente investigación se encontró que las madres se dedican exclusivamente al cuidado de sus hijos, por sentirse responsables ante sus familias y la sociedad. Con el transcurrir de los primeros meses de nacido, sienten un vínculo tan fuerte que consideran que no se deben separar de ellos por temor a que algo malo les suceda y se sientan con la responsabilidad de evitar que se les repita la misma historia de ellas. Las madres adolescentes, en la presente investigación, se mostraron interesadas por educar de mejor manera a sus hijos, y muestran deseo de ser buenas madres y tener confianza con sus hijos. Se encontró que sienten que no podrían separarse de ellos y que deben dar a ellos lo mejor de sí.

Respecto al valor que otorgan las madres adolescentes a sus hijos, en este trabajo se encontró que los conciben como el eje central de su vida, el motor que les permite salir adelante; los consideran como lo único propio y una compañía real para toda la vida, lo cual confirma lo propuesto Peña, Villavicencio, Palacios \& Mora (2015) de que, a pesar 
de referir y/o aplicar un modelo de crianza heredado o permisivo, establecen una ruta de crianza en el hijo, que adopta la premisa de realizar lo que la madre no hizo en su edad, o superar los limitantes que tuvo la madre en su momento y que no desea se presenten en el hijo.

De cierta manera, las madres adolescentes perciben a su hijo como una manera de construir su propia vida y sienten poder sobre ellos; el poder de poder guiar y orientar la vida de sus hijos de la manera que ellas consideren apropiada; buscan que sus hijos las reconozcan como autoridad, se preocupan por ganarse su respeto. La madres, por lo general, "idealizan" el proceso de crianza que quieren tener con sus hijos, considerando que debe ser distinto a los patrones de crianza que ellos recibieron de sus familias, lo cual coincide con la afirmación que hace Coronado (2013) de que las madres adolescentes le dan resignificaciones a la disciplina con las que fueron educadas y hacen de manera consciente cambios de estrategias en la manera de sancionar o castigar a sus hijos: prefieren el diálogo y evitan el castigo físico. Sin embargo, en la presente investigación se encontró cómo se puede confirmar en los resultados que las madres reconocen la importancia del diálogo y evitan el maltrato físico; aunque sienten necesidad de cambiar las prácticas con las que fueron criadas, en la realidad presentan una tendencia a replicar muchos de esos patrones, e incluso piden apoyo y asesoría a sus familiares y/o cuidadores más cercanos.

De igual forma, otro aspecto de gran importancia encontrado en la presente investigación es coherente con la propuesta de Peña, Villavicencio, Palacios \& Mora (2015), en el sentido de que las madres adolescentes primerizas adoptan los modelos de crianza con los que fueron criadas; pueden aplicar el modelo de crianza del nuevo entorno familiar de su pareja o pueden establecer según sus preceptos uno que vaya en contravía del recibido, dado que piensan que les representa desventajas en términos de emociones y emancipación. En esta investigación, se evidencia que las madres sienten temor y miedo al no poder identificar síntomas de problemas de salud, necesidades emocionales y fisiológicas. Las madres se muestran con gran interés de satisfacer, de manera inmediata, las necesidades de sus hijos; se muestran consentidoras y, cuando las solicitudes requieren hacer gastos económicos para los cuales no están en condiciones, muestran sentimiento de tristeza; frente a ello recuren al diálogo y a explicarles por qué no pueden complacerlos.

En suma, se encontró que el vínculo afectivo de las madres adolescentes con sus hijos se fortalece debido al apoyo o respaldo que encuentren en sus familias, dentro de los cuales predomina el apoyo de otras mujeres de la familia, quienes actúan como modelo en términos de cuidados y experiencia en los procesos de crianza. De manera paralela, como se fortalece el vínculo afectivo con los niños también se fortalece el vínculo con las figuras femeninas que las apoyen. En la presente investigación se encontró que la relación familiar -en especial con figuras femeninas, como madres, suegras, abuelas y tías- mejora de manera significativa, ya que aumenta la comunicación, el tiempo de interacción y el agradecimiento por el apoyo en el nuevo rol. Esto es coherente con los aportes de Barrero (2010), quien también reconoce la importancia del rol de las abuelas dentro del proceso de apoyo y crianza de los hijos de madres adolescentes, ya que afirma que, en su mayoría, viven en sistemas familiares de tres generaciones, conformados por abuelos, madre adolescente y el bebé. Sin embargo, el mayor apoyo se da por partes de las figuras femeninas; en cambio las figuras masculinas, como padres 
y/o cuidadores, muestran mayor resistencia a la aceptación del bebé y se muestran un poco indiferentes a brindar apoyo económico y emocional.

Según Nieto et al. (2012), para las madres adolescentes el apoyo percibido de la pareja es un punto de gran importancia en el proceso de racionalización del embarazo. Se considera que tienen buena expectativa de la reacción del novio y esta tiene mucho peso en cualquier decisión. Las jóvenes perciben que tener el apoyo de sus parejas se traduce o es un paso importante para que se dé una aceptación social de su embarazo, mientras que, en la presente investigación, se encontró que, aunque para las adolescentes es importante el apoyo de su pareja, ellas consideran a su hijo como algo propio, exclusivo de ellas. No relacionan mucho el apoyo de su pareja con la disminución de la presión social, ya que sienten que esta se da más por su edad, ya que no está bien visto que una adolescente quede en estado de embarazo; aunque son agradecidas con el apoyo de sus parejas, en los casos que no lo reciben se sienten capaces de enfrentar solas la situación. Dentro de este trabajo, se ha considerado pertinente proponer este tema para futuras investigaciones, ya que, por los cambios sociales, se han evidenciado cambios en la percepción de los adolescentes frente a la importancia del apoyo de una pareja en el embarazo adolescente y en su proceso de aceptación social, ya que algunas veces le dan más valor a estar solas que con parejas que, socialmente, no son bien vistas.

En adición, el estudio también ha permitido hacer un acercamiento a la comprensión de la percepción de las jóvenes frente a los cambios sociales, retos y nuevos desafíos a los que se enfrentan al ser madres adolescentes. Como se mencionaba anteriormente, en el plano familiar se fortalecen las relaciones y vínculos; sin embargo, en el plano social o de interacción con pares o personas diferentes a la familia, se evidencia deterioro o fracturación, ya que toman distancia de sus pares al momento que se retiran del colegio o dejan de frecuentarlos debido a que los intereses de ambas partes son distintos: los otros continúan en sus planes de salidas, paseos, rumba; y ellas, esmeradas o preocupadas por cumplir su rol de madres, toman distancia de toda su vida o rutinas anteriores. De cierta manera, las madres adolescentes se sienten diferentes a sus pares, e incluso terminan alejándose, al considerar que ellas deben pagar las consecuencias de sus actos, y una de ellas es dejar su vida de jóvenes y dedicarse exclusivamente a los cuidados de su hijo. La fracturación de relaciones sociales o la no participación en grupo de interacción social distintos a las familias, hace que las jóvenes adolescentes se sientan aisladas y, a su vez, ello dificulte el crecimiento educativo y laboral. Las jóvenes terminan inmersas solo en su mundo familiar, $y$, al no interactuar con pares o adultos de otros ámbitos, pierden el interés por crecer en otros aspectos de su vida. Se podría decir que la falta de interacción y el aislamiento se convierte en un factor de riesgo, complejiza aún más la situación y disminuye las posibilidades de superarla de manera satisfactoria y mejorar las condiciones de vida familiar. Todo lo anterior, sumado a lo que plantean Rojas, Alarcón \& Calderón (2010), quienes encontraron en su investigación que el embarazo adolescente si afecta la relación con sus amigos, compañeros o pares ya que en la mayoría de casos se encontró que sentían vergüenza frente a ellos y, por tal razón, intentaron ocultárselos.

En la presente investigación se encontró que las madres adolescentes socializan muy poco con los adultos de su contexto social, ya que consideran que son vistas de manera distinta y se alejan de ellos con el fin de evitar sentirte cuestionadas o reprochadas. En estos contextos sociales sienten que son objeto de burla, de reproche y críticas, lo 
cual, finalmente, termina en toma de distancia de los círculos sociales y se inmersa por completo en el seno de su hogar, cuidados de sus hijos; y evitan satisfacer, incluso sus propias necesidades psicológicas y sociales, por temor a que cuestionen su rol o responsabilidad de madre. En este momento todas las actividades de socialización giran en torno a su hijo. En la situación de las madres adolescentes se debe considerar la importancia de las redes de apoyo, ya que ellas manifiestan la importancia de sentirse aceptadas y no desprestigiadas, como consecuencia de su embarazo. Ellas se muestran muy agradecidas con las personas que las apoyan, y se presume que las redes de apoyo adicionales a la familia pueden ayudar a la superación de la situación.

\section{REFERENCIAS}

Barrero, M. J. O. (2010). Las abuelas como cuidadoras: una visión ecológica de su rol. Revista de Investigaciones UNAD, 9(1), 225-240.

Bensaja, E., Garcia, A., Neiva, L., Nieto, C., Koller, S. (2012). Características familiares y apoyo percibido entre adolescentes brasileños con y sin experiencia de embarazo. Universidad Federal Do Rio Grande.

Carrillo, S., Maldonado, C., Saldarriaga, L. M., Vega, L., Díaz, S. (2004). Patrones de apego en familias de tres generaciones: abuela, madre adolescente, hijo. Revista Latinoamericana de Psicología, 36(3), 409-430.

Coronado Mendoza, A. M., Ortiz Gómez, N. E. (2013). Rol materno y pautas de crianza en nueve madres adolescentes desde una perspectiva generacional, pertenecientes a las UPA de la Fundación Carla Cristina.

De Castro, A. (2003). Introduction to Giorgi's existential phenomenological research method. Psicología desde el Caribe. Universidad del Norte.

De Castro. A. (2005). A Hermeneutical Understanding of Experience of Being Anxious. Journal Qualitative Research in Psychology, 2(2), 141 - 167.

Departamento Administrativo Nacional de Estadística. (2015). https://www.dane.gov. co/files/investigaciones/poblacion/cifras-definitivas-2015.pdf

Echevarría, M. (2015). Depresión postparto en Madres Adolescentes. Universidad de la Rioja.

Gago, J. (2013). Teoría del apego. El vínculo Escuela Vasco Navarra de Terapia Familiar. http://www.avntf-evntf.com/wp-content/uploads/2016/06/Teoría-del-apego.-Elvínculo.-J.-Gago-2014.pdf 
Main, M., Solomon, J. (1986). Discovery of an insecure-disorganized/disoriented attachment pattern. In T. B. Brazelton \& M. W. Yogman (Eds.), Affective development in infancy ( $\mathrm{p}$. 95-124). Ablex Publishing.

Mazuera, R. Trejos, A., Reyes, L. (2017). Percepción del embarazo adolescente en el Departamento Norte de Santander. Rev. Salud Pública. 19(6): 733-738.

Nieto, C. Á., Moreno, G. P., Abad, M. L., Martos, J. S., Olalla, L. R. (2012). Motivaciones para el embarazo adolescente. Gaceta Sanitaria, 26(6), 497-503. https://www. sciencedirect.com/science/article/pii/S0213911112000878

Ortega, A., Rodríguez, D., Jiménez, A. (2013). Equilibrio trabajo-familia: corresponsabilidad familiar y autoeficacia parental en trabajadores de una empresa chilena. Diversitas: Perspectivas en Psicología, 9(1), 55-64.

Peña, P., Villavicencio, L., Palacios, M., Mora, J. (2015). Maternidad adolescente y estilo de crianza actual. Acción pedagógica, 24, 62 - 72.

Profamilia. (2010) Encuesta nacional de demografía y salud. Tomo II. https://profamilia. org.co/wp-content/uploads/2019/05/ENDS-2015-TOMO-II.pdf

Rojas García, D. C. P.; Alarcón Ovalle, M. H. ; Calderón, B. P. (2010). Vivencia del Embarazo en Adolescentes en una institución de protección en Colombia. Index de Enfermería, 19 (2-3), 129-133. http://scielo.isciii.es/scielo.php?script=sci arttext\&pid=S1132$12962010000200012 \& \operatorname{lng}=$ es\&tlng=es

Secretaria de salud de Barranquilla. (2015). Plan de salud territorial distrito de Barranquilla. Análisis de la situación de salud-capitulo II. https://www.minsalud. gov.co/plandecenal/mapa/analisis-de-Situacion-Salud-Barranquilla-2012-2015.pdf

Vélez Arango, A. L. (2012). El embarazo en el adolescente: una visión desde la dimensión emocional y la salud pública. Revista Cuidarte, 3(1).

Vélez, E., Figueredo, L. (2015). Impacto psicosocial del embarazo en las adolescentes. Revista Educación en Valores, 1(23), 18. http://servicio.bc.uc.edu.ve/ multidisciplinarias/educacion-en-valores/v1n23/vol23n12015.pdf\#page=24 